\title{
On Surface Oxidation Issues Arising during CO Oxidation on a PdAu(110) Surface. An In Situ Study by Complementary Environmental Methods (STM, SXRD, XPS)
}

Marie-Angélique Languille ${ }^{1,2}$, Eric Ehret ${ }^{1}$, Marie-Claire Saint-Lager ${ }^{3}$, Aude Bailly ${ }^{3}$, Bongjin Simon Mun $^{4,5}$, Jean-Jacques Gallet ${ }^{6}$, Fabrice Bournel ${ }^{6}$, and Francisco J. Cadete Santos Aires ${ }^{1,7}$

${ }^{1}$ IRCELYON, UMR 5256 CNRS, Univ. Claude Bernard Lyon 1, 69626 Villeurbanne cedex, France.

${ }^{2}$ Present address: CRCC/CRC, USR 3224, CNRS, MNHN, Ministère Culture, 75005 Paris, France.

${ }^{3}$ Institut Néel, UPR 2940, CNRS, Sorbonne Univ., 38042 Grenoble cedex 9, France.

${ }^{4}$ Department of Physics and Photon Science, GIST, Gwangju, 61005, Republic of Korea.

${ }^{5}$ Center for Advanced X-ray Science, GIST, Gwangju, 61005, Republic of Korea.

${ }^{6}$ LPCMR, UMR 7614, CNRS, Sorbonne Univ., 75252 Paris Cedex 05, France.

${ }^{7}$ LCR, National Research Tomsk State University, Tomsk 634050, Russian Federation.

$\mathrm{CO}$ oxidation is generally performed with an excess of oxygen in the reactive gas mixture. Whether this is a low excess $\left(\mathrm{O}_{2} / \mathrm{CO}\right.$ ratio in the 2 to 5 range often used in catalytic tests over realistic powder supported catalysts) or much higher excess $\left(\mathrm{O}_{2} / \mathrm{CO}\right.$ ratio in the 10 to 1000 range used in more model approaches) the question of surface oxidation and its role in the catalytic reaction is thus relevant. With these issue in mind, we have studied in situ the evolution and catalytic performance of the $\mathrm{Pd}_{70} \mathrm{Au}_{30}(110)$ in $\mathrm{CO}$ oxidation by complementary surface science methods operating in quasi-realistic catalytic conditions (variable T and $\mathrm{P}_{\text {gas }}$ ): NAP-XPS and Environmental STM and SXRD. The STM is a modified, gold-plated, OMICRON instrument operated within a home-made vacuum/pressure system [1,2]. The SXRD environmental system was developed by M.C. Saint-Lager et al to be operated in the ESRF BM32 beamline [3,4]. Both systems are capable of operating over 12 decades of gas pressure $\left(10^{-9}-10^{3} \mathrm{mBar}\right)$ and variable temperature. Two NAP-XPS systems were operated in gas pressures up to $20 \mathrm{mBar}$ and variable temperature: one system is installed at ALS in beamline 9.3.2. [2,5], the other system is installed at SOLEIL in beamline TEMPO [6]. The $\mathrm{Pd}_{70} \mathrm{Au}_{30}(110)$ (from SPL) is an unreconstructed $\mathrm{Au}$-rich ( 8590\%) surface under stable conditions after preparation cycles (ion bombardment followed by annealing). We have shown that upon exposure to $\mathrm{CO}, \mathrm{Pd}$ segregates to the surface and the surface becomes rough (corrugation $\sim 0.05 \mathrm{~nm}$ ) [4]. In presence of oxygen, the surface reconstructs exhibiting a (nx2) structure ((1x2) with vacancies) and, as for CO, Pd segregates to the surface (Fig.1). Increasing the temperature and/or oxygen pressure eventually leads to oxidation of the Pd-rich surface. Under reaction conditions, for $\left.\mathrm{P}\left(\mathrm{O}_{2}\right) / \mathrm{P}(\mathrm{CO}) \approx 10\right)$, NAP-XPS reveals that the reaction occurs in the presence of adsorbed oxygen species at the surface but that the activity strongly decreases when oxidation of the surface appears at higher $\mathrm{T}$ (Fig.2). In the case of $\mathrm{P}\left(\mathrm{O}_{2}\right) / \mathrm{P}(\mathrm{CO})>>10$, SXRD shows that an oxide strained layer $(<10$ atomic layers) is formed (Fig.3) that is the active surface in such conditions [4]. Surface oxidation and its role in the $\mathrm{CO}$ oxidation strongly depend on the reaction conditions and for a same system surface oxidation can be either noxious or the best option to the reaction.

References:

[1] Y Jugnet et al, Surface Science 521 (2002) L639.

[2] MA Languille et al, Catalysis Today 260 (2016) 39.

[3] MC Saint-Lager et al, Journal of Physical Chemistry C 122 (2019) 22588.

[4] MC Saint-Lager et al, ACS Catalysis 9 (2019) 4448.

[5] Y Jugnet et al, Journal of Physical Chemistry Letters 3 (2012) 3707. 
[6] H Liu et al, ACS Catalysis 9 (2019) 10212.

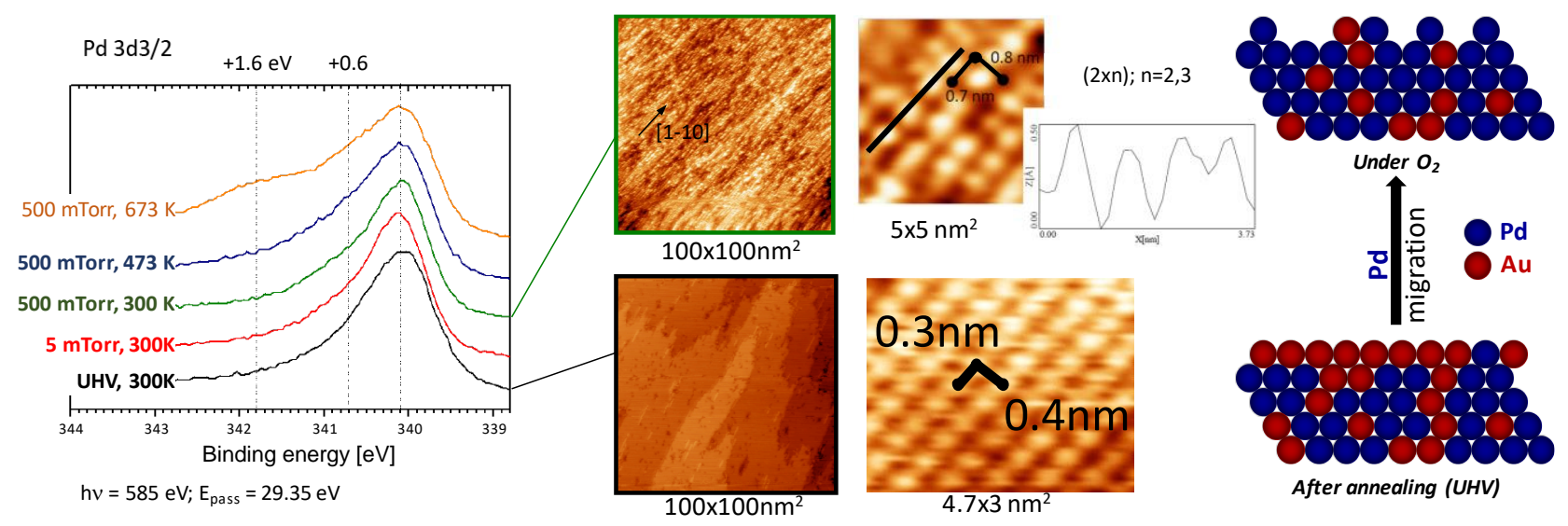

Figure 1. The PdAu(110) surface upon exposure to oxygen: (left) appearance of a high binding energy shoulder on Pd3d $\mathrm{d}_{3 / 2}$ XPS peak with increasing oxygen pressure and temperature; (center) reconstruction of the surface as seen by STM and Pd segregates to the surface; (right) schematics of the evolution.
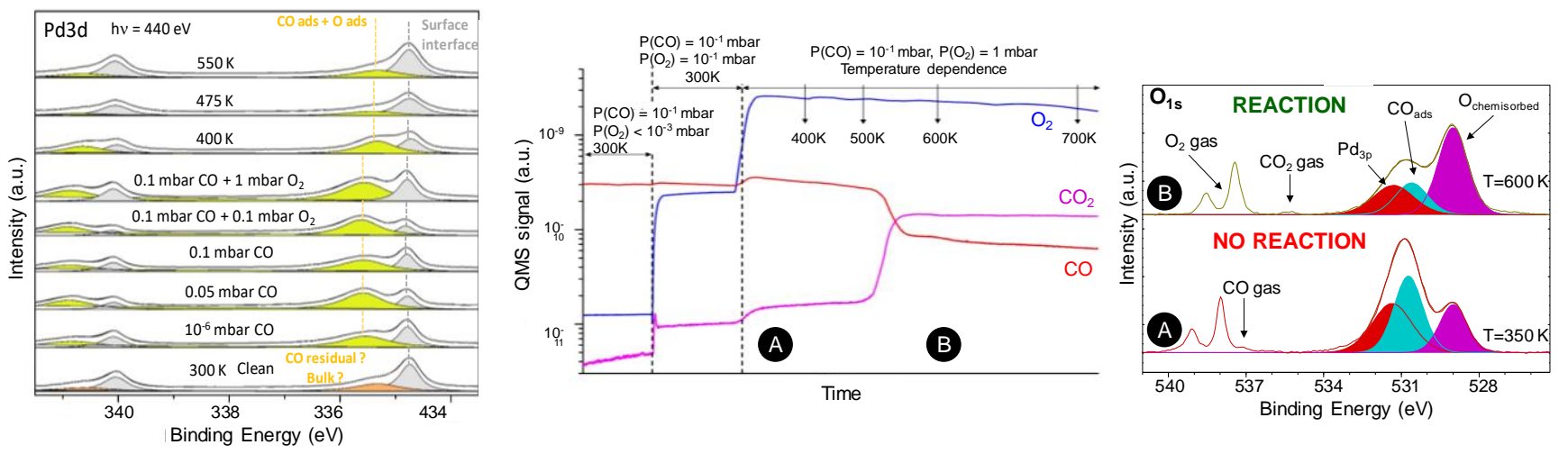

Figure 2. (left) XPS Pd3d peak evolution with gas mixture, pressure and temperature; (center) QMS and (right) corresponding XPS O1s peaks (solid and gas phases) for active and inactive conditions.
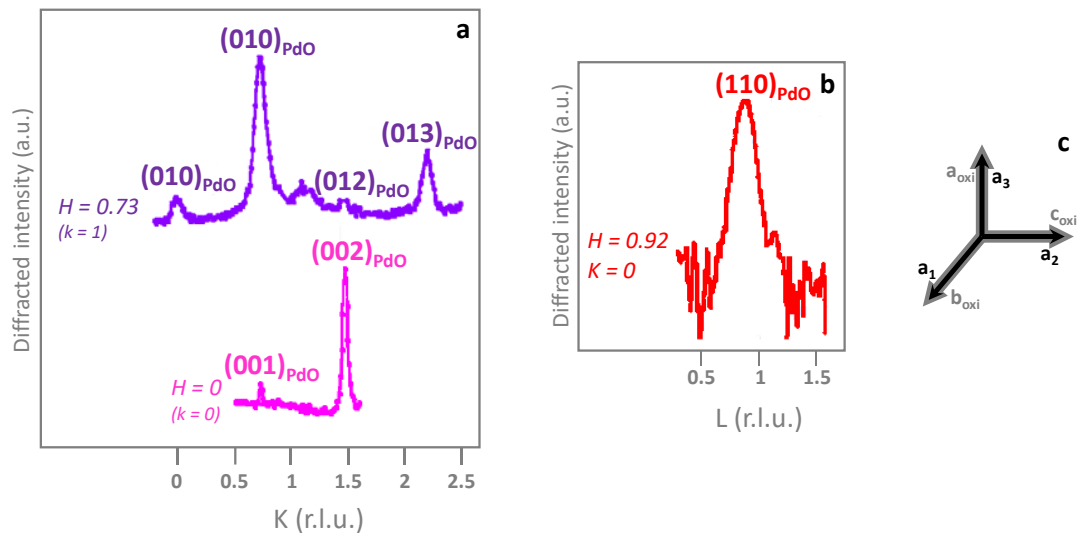

Figure 3. $\mathrm{PdO}$ peaks measured by SXRD along several directions of the reciprocal space (along $K$ in with $L=0$ and $H=0$ and $H=0.73$, in a; along $L$ with $H=0.92$ and $K=0$, in $\mathbf{b}$ ) in the frame of the fcc $\operatorname{PdAu}(110)$ surface by $\left(\mathrm{a}_{1}, \mathrm{a}_{2}, \mathrm{a}_{3}\right)$ with the Miller indices $(H, K, L)$ and the oxide peaks are labeled with the Miller indices $(h, k, l)$ in the $\mathrm{PdO}$ frame $\left(\mathrm{a}_{\mathrm{oxi}}, \mathrm{b}_{\mathrm{oxi}}, \mathrm{c}_{\mathrm{oxi}}\right)$ defined in $\mathbf{c}$. 